\title{
Flore et structure de la végétation ligneuse le long de la Grande Muraille Verte au Ferlo, nord Sénégal.
}

\author{
Khoudia NIANG1, Ousmane NDIAYE¹, Aly Diallo et Aliou GUISSE1;2 * \\ 1 Université Cheikh Anta DIOP BP 5005 Fann - Dakar (Sénégal)/Faculté des Sciences et Techniques/Département \\ de Biologie végétale/Laboratoire d'Écologie et d'Ecohydrologie. \\ 2 Observatoire Homme-Milieu (OHMi) Tessékéré, et UMI 3189 \\ *Auteur correspondant. E-mail: aliou.guisse@ucad.edu.sn ou alguisse@orange.sn, Tel. (+221) 776389669
}

Original submitted in on 28 th March 2014. Published online at www.m.elewa.org on $31^{\text {st }}$ July 2014. http://dx.doi.org/10.4314/jab.v79i1.15

\section{RESUME}

Objectif: Ce travail réalisé dans les parcours communautaires du Ferlo-Nord, le long du tracé de la Grande Muraille Verte, a pour objectif d'étudier les caractéristiques essentielles de la végétation ligneuse pouvant servir de référence à l'étude de sa dynamique ou toute autre étude nécessitant un état des lieux par rapport à la végétation.

Méthodologie et résultats: La méthode d'échantillonnage par relevés dendrométriques utilisée, a permis d'obtenir 20 espèces appartenant à 18 genres et 12 familles. La famille des Mimosaceae est la mieux représentée, suivie de celles des Combretaceae, Asclepiadaceae et Papilionaceae. La distribution selon la hauteur et selon la circonférence du tronc à $30 \mathrm{~cm}$ du sol des individus du peuplement montre une dominance de la strate arbustive. La régénération plus importante à Widou est dominée par Balanites aegyptiaca, Calotropis procera et Boscia senegalensis qui semblent être plus adaptées aux conditions climatiques sévères et aux actions anthropiques croissantes.

Conclusion: Cette étude a mis en évidence la régression de certaines espèces ligneuses à intérêt multiple pour la population locale du Ferlo-nord et l'expansion d'autres moins utilisées. Ainsi, dans le cadre de la Grande Muraille Verte par rapport aux conditions du milieu (les aléas climatiques et les usages multiples par les populations locales), le choix d'espèces adaptées et à protéger devra être guidé par cette présente étude.

Mots clés : Ferlo, structure, Flore, Ligneux.

\section{Nature and structure of woody vegetation along the Great Green Wall in the Ferlo region of Northern Senegal.}

Objective: The work done in the Ferlo North region,along the Great Green Wall aims to study the essential characteristics of woody vegetation in order to serve as a reference for the study of dynamics in other study areas.

Methodology and results: the sampling method using dendrometric surveys indicated the presence of 20 species belonging to 18 genera and 12 families. The Mimosaceaefamily is the most highly represented, followed by the Combretaceae, Asclepiadaceae and Papilionaceae. Population distribution based on the height and the circumferences of trunks at $30 \mathrm{~cm}$ above soil level indicated a predominance of shrubs. The greatest 


\section{Niang et al. J. Appl. Biosci. 2014. Flore et structure de la vegetation ligneuse le long de la Grande}

Muraille Verte au Ferlo, nord Senegal

regeneration potential was observed in Widou where Balanites aegyptiaca, Calotropis Procera and Boscia senegalensis dominated the landscape. These species are highly adapted to harsh climatic conditions and increasing human activities.

Conclusion: This study showed the regression of some useful multi-purpose woody species for the local populations of North Ferlo whereas other less valuable species became dominant in the landscape. Thus, in the context of the Great Green Wall, this study is particularly useful in order to determine the choice of tree species for reforestation and protection.

Keywords : Ferlo, structure, Flora, Trees.

\section{INTRODUCTION}

Au Sahel et en particulier au Ferlo, les actions combinées de la péjoration climatique et de l'action anthropique aboutissent à la dégradation de la végétation (Grouzis et Albergel, 1989; Diouf et al., 2002; Niang, 2009). Les aléas climatiques se traduisent par une pluviométrie faible et irrégulière, tandis que l'action anthropique se résume par l'utilisation des ligneux comme fourrage (Le Houerou, 1980; Diouf et al., 2002; Niang, 2009). En effet, dans cette zone, le tapis herbacé ne subsiste que pendant quelques mois; il devient rare pendant la saison sèche et présente une valeur nutritive assez faible (Akpo et al., 1995). A cette période de l'année la végétation ligneuse reste la principale source de fourrage donc un complément alimentaire

\section{MATERIEL ET METHODE}

Zone d'étude : La zone d'étude appartient au Ferlo sableux caractérisé par une succession de dunes et de bas-fonds peu accidentés. Elle se situe à la région de Louga et est limitée à l'est par la région de Matam et au nord par celle de Saint-Louis (Fig. 1). Son climat est caractérisé par l'alternance de deux saisons: une saison sèche de 9 mois (octobre à juin) et une saison pluvieuse de 3 mois. La pluviométrie reste faible et très instable avec une moyenne de $422,6 \mathrm{~mm}$ par an pour un coefficient de variation de 0,3 sur la période 1951-2004 intéressant pour le bétail. Or l'élevage qui contribue au Sénégal jusqu'à hauteur de $6,5 \%$ au produit intérieur brut $(\mathrm{PIB})$, représente 55 à $70 \%$ des revenus ruraux (P.O.D.E.S., 2004). II constitue l'activité principale de la zone d'étude. C'est ainsi que nous nous sommes fixés comme objectif principal de caractériser cette végétation afin d'obtenir une base de données solide avant toute autre action ultérieure. Notre argumentation se fonde sur deux hypothèses: une diminution de certaines espèces (Poupon et Bille, 1974; Sharman et Gning, 1983; Toutain et al., 1983; Diouf et al., 2002; Ndiaye, 2013) et une forte dégradation des écosystèmes sahéliens en raison de la péjoration des conditions climatiques et de l'anthropisation croissante.

(Faye et al., 2011). La température moyenne oscille autour de $27,73^{\circ} \mathrm{C}$ et fluctue entre une moyenne maximale de $30,19^{\circ} \mathrm{C}$ au mois d'octobre et minimale de $24,48^{\circ} \mathrm{C}$ au mois de janvier (Niang, 2009). L'étude a été menée en 2009 dans quatre stations: Widou-Thiengoly, Tessékéré Forage, Labgar et Lougré-Thiolly (Fig. 1). Leur choix se justifie par l'importance de l'action anthropique liée au surpâturage, le passage du tracé de la Grande Muraille Verte (GMV) et l'influence des différents projets et observatoires installés dans ces sites. 


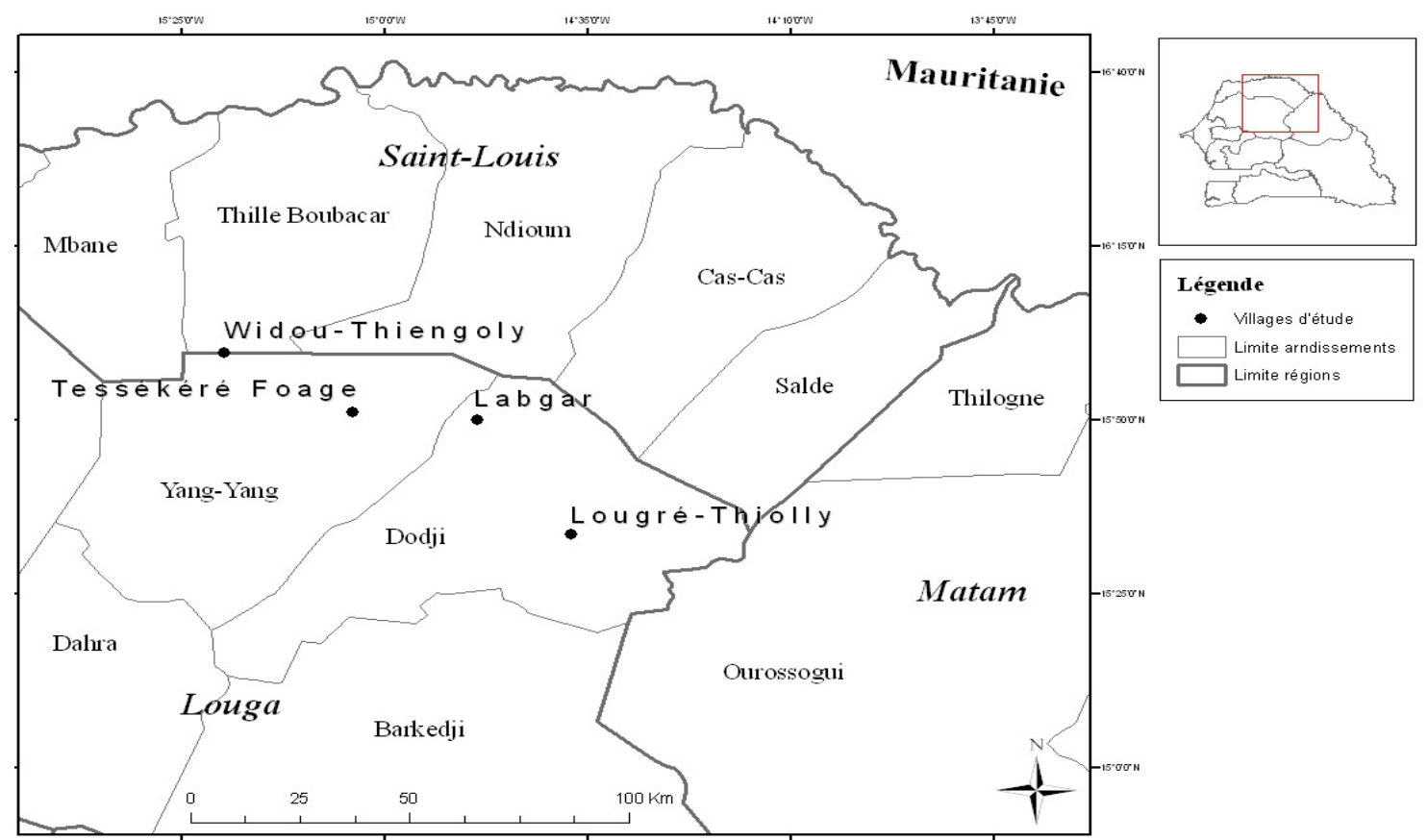

Figure1 : Localisation des sites dans la carte de situation du Sénégal

\section{Méthode}

Collecte de données : Pour connaitre l'état actuel de la végétation ligneuse, nous avons procédé par un échantillonnage et par des relevés dendrométriques. Ainsi, nous avons réalisé 60 relevés de $2500 \mathrm{~m}^{2}$ (Boudet, 1984), en raison de 15 relevés par site. Dans chaque placette, l'identification des individus dont la circonférence à la base est supérieure à $10 \mathrm{~cm}$, a été réalisée, puis des mesures dendrométriques (circonférence du tronc à $30 \mathrm{~cm}$ du sol, diamètres du houppier et hauteur totale) ont été effectuées. La distance entre les individus a été aussi mesurée par la méthode du plus proche arbre et les individus dont la circonférence à la base est inférieure à $10 \mathrm{~cm}$ sont comptés pour l'évaluation de la régénération.

Traitement des données : La fréquence de présence est calculée à partir de la formule $\mathbf{F i}=\frac{\mathbf{N r i}}{\mathrm{Nr}} \times \mathbf{1 0 0}$ avec $\boldsymbol{F}_{i}=$ fréquence de présence exprimée en

\section{RESULTATS}

Composition de la flore : La flore du Ferlo à travers les quatre sites étudiés est riche de 20 espèces appartenant à 18 genres et à 12 familles. Chaque famille est représentée par un genre et une espèce à l'exception de la famille des Asclepiadaceae et Papilionaceae (2 genres pourcentage (\%); $\boldsymbol{N}_{r i}=$ nombre de relevés où l'on retrouve l'espèce $\boldsymbol{i} ; \boldsymbol{N}_{r}=$ nombre total de relevés. La densité relative est obtenue à partir de la formule suivante: $D_{r}=\frac{N_{i}}{N} \times 100$ avec $D r=$ densité relative exprimée en pourcentage (\%); $\mathrm{Ni}$ = l'effectif de l'espèce de rangi; $\mathrm{N}$ $=$ l'effectif total de toutes les espèces confondues.

La circonférence à $30 \mathrm{~cm}$ du sol (C) et le diamètre du houppier (D) ont permis d'évaluer les recouvrements basal et aérien respectivement par la surface terrière (St) et celle de la couronne (Sc) (Matern, 1956). Ils permettent aussi d'étudier la structure horizontale de la végétation.

$S t=\sum \frac{C^{2}}{4 \pi} \quad S c=\sum \frac{\pi D^{2}}{4}$. La hauteur des arbres permet d'étudier la structure verticale de ce peuplement à travers la distribution fréquentielle des individus selon leur hauteur.

et 2 espèces), des Combretaceae ( 3 genres et 3 espèces) et les Mimosaceae ( 3 genres et 5 espèces) (Tableau 1). 
Tableau 1 : La flore inventoriée en fonction des différents sites.

\begin{tabular}{|c|c|c|c|c|c|}
\hline Familles & 1 & 2 & 3 & 4 & Espèces \\
\hline Anacardiaceae & + & + & & + & Sclerocarya birrea (A. Rich.) Hochst. \\
\hline Apocynaceae & + & & + & & Adenium obesum (Forsk.) Roem.et Sc. \\
\hline \multirow[t]{2}{*}{ Asclepiadaceae } & + & + & + & & Calotropis procera Ait. \\
\hline & + & & & & Leptadenia pyrotechnica (Forsk.) Decne. \\
\hline Balanitaceae & + & + & + & + & Balanites aegyptiaca (L.) Del. \\
\hline Bignoniaceae & & & & + & Kigelia africana (Lam.) Renth. \\
\hline Bombacaceae & + & + & + & + & Adansonia digitata $\mathrm{L}$. \\
\hline Capparidaceae & + & + & + & & Boscia senegalensis (Pers.) Lam. \\
\hline \multirow[t]{3}{*}{ Combretaceae } & & + & & + & Anogeissus leiocarpa (DC.) G.et Perr. \\
\hline & + & & & + & Combretum glutinosum Perr. \\
\hline & + & & & + & Guiera senegalensis J.F.Gmel \\
\hline \multirow[t]{5}{*}{ Mimosaceae } & + & + & & + & Acacia senegal (L.) Willd. \\
\hline & + & + & + & + & Acacia seyal Del. \\
\hline & + & + & + & + & Acacia tortilis (Forst.) Hyane subsp. raddiana \\
\hline & & & & + & Dichrostachys glomerata (Forsk).C.Chr. \\
\hline & & & + & & Prosopis chilensis (Mol.) Stuntz. \\
\hline \multirow[t]{2}{*}{ Papilionaceae } & & & + & & Dalbergia melanoxylon G. et Perr \\
\hline & & & & + & Pterocarpus lucens Lepr. \\
\hline Rhamnaceae & + & + & + & & Ziziphus mauritiana Lam. \\
\hline Tiliaceae & + & & + & + & Grewia bicolor Juss. \\
\hline
\end{tabular}

(1 =Tessékéré forage; 2 =Widou-Thiengoly; 3=Labgar; 4=Lougré-Thiolly;+= présence).

Caractéristiques structurales : Les 5 espèces les plus fréquentes de la zone étudiée sont: $B$. aegyptiaca, $A$. tortilis $B$. senegalensis, A. senegal et $C$. procera. Elles sont respectivement rencontrées dans $83 \%, 43 \%, 35 \%$ et $27 \%$ des placettes ou relevés. $B$. aegyptiaca, l'espèce la plus fréquente de la zone est rencontrée dans $100 \%$ des placettes à Widou et à Labgar, dans $93 \%$ des placettes à Lougré et dans $40 \%$ des placettes à Tessékéré (Tableau 2). Notons le cas de $A$. digitata qui garde la même fréquence dans tous les sites et se rencontre partout dans $7 \%$ des placettes.

Tableau 2 : Variation de la fréquence de présence et de la densité relative de différentes espèces ligneuses rencontrées dans les différents sites

\begin{tabular}{lcccccc}
\hline \multirow{2}{*}{ Espèces } & \multicolumn{7}{c}{ Fréquences de présence (\%) } & densité \\
\cline { 2 - 7 } & Total & $\mathbf{1}$ & $\mathbf{2}$ & $\mathbf{3}$ & $\mathbf{4}$ & \\
\hline Acacia senegal (L.) Willd. & 35 & 13 & 47 & 0 & 80 & 9.40 \\
\hline Acacia seyal Del. & 12 & 7 & 27 & 7 & 7 & 1.96 \\
\hline Acacia tortilis (Forst.) Hyane subsp. raddiana & 43 & 7 & 87 & 40 & 40 & 5.68 \\
\hline Adansonia digitata L. & 7 & 7 & 7 & 7 & 7 & 0.49 \\
\hline Adenium obesum (Forsk.) Roem.et Sc. & 3 & 7 & 0 & 7 & 0 & 0.20 \\
\hline Anogeissus leiocarpa (DC.) G.et Perr. & 3 & 0 & 0 & 0 & 13 & 0.69 \\
\hline Balanites aegyptiaca (L.) Del. & 83 & 40 & 100 & 100 & 93 & 47.50 \\
\hline Boscia senegalensis (Pers.) Lam. & 35 & 15 & 7 & 33 & 0 & 12.54 \\
\hline Calotropis procera Ait. & 27 & 80 & 27 & 0 & 0 & 12.83 \\
\hline
\end{tabular}




\begin{tabular}{|c|c|c|c|c|c|c|}
\hline $\begin{array}{l}\text { Niang et al. J. Appl. Biosci. } 2014 . \\
\text { Muraille Verte au Ferlo, nord Senegal }\end{array}$ & $e \mathrm{de}$ & & & & & \\
\hline Combretum glutinosum Perr. & 12 & 7 & 7 & 0 & 33 & 1.86 \\
\hline Dalbergia melanoxylon G. et Perr. & 3 & 0 & 0 & 13 & 0 & 0.39 \\
\hline Dichrostakys glomerata (Forsk).C.Chr. & 2 & 0 & 0 & 0 & 7 & 0.29 \\
\hline Grewia bicolor Juss. & 10 & 7 & 0 & 13 & 20 & 0.98 \\
\hline Guiera senegalensis J.F.Gmel & 7 & 13 & 0 & 0 & 13 & 0.39 \\
\hline Kigelia africana (Lam.) Renth. & 2 & 7 & 0 & 0 & 0 & 0.10 \\
\hline Leptadenia pyrotechnica (Forsk.) Decne. & 7 & 27 & 0 & 0 & 0 & 0.69 \\
\hline Prosopis chilensis (Mol.) Stuntz.) & 2 & 0 & 0 & 7 & 0 & 0.10 \\
\hline Pterocarpus lucens Lepr. & 3 & 0 & 0 & 0 & 13 & 0.20 \\
\hline Sclerocarya birrea (A. Rich.) Hochst. & 18 & 40 & 27 & 0 & 7 & 2.64 \\
\hline Ziziphus mauritiana Lam. & 13 & 13 & 13 & 27 & 0 & 1.08 \\
\hline
\end{tabular}

. (1 =Tessékéré forage; $\mathbf{2}$ =Widou-Thiengoly; $\mathbf{3}$ =Labgar; 4 =Lougré-Thiolly)

Structure du peuplement: La structure verticale évaluée à partir de la distribution selon la hauteur des individus du peuplement $(A)$ et des quatre espèces dominantes (B) est représentée par la Figure 2.

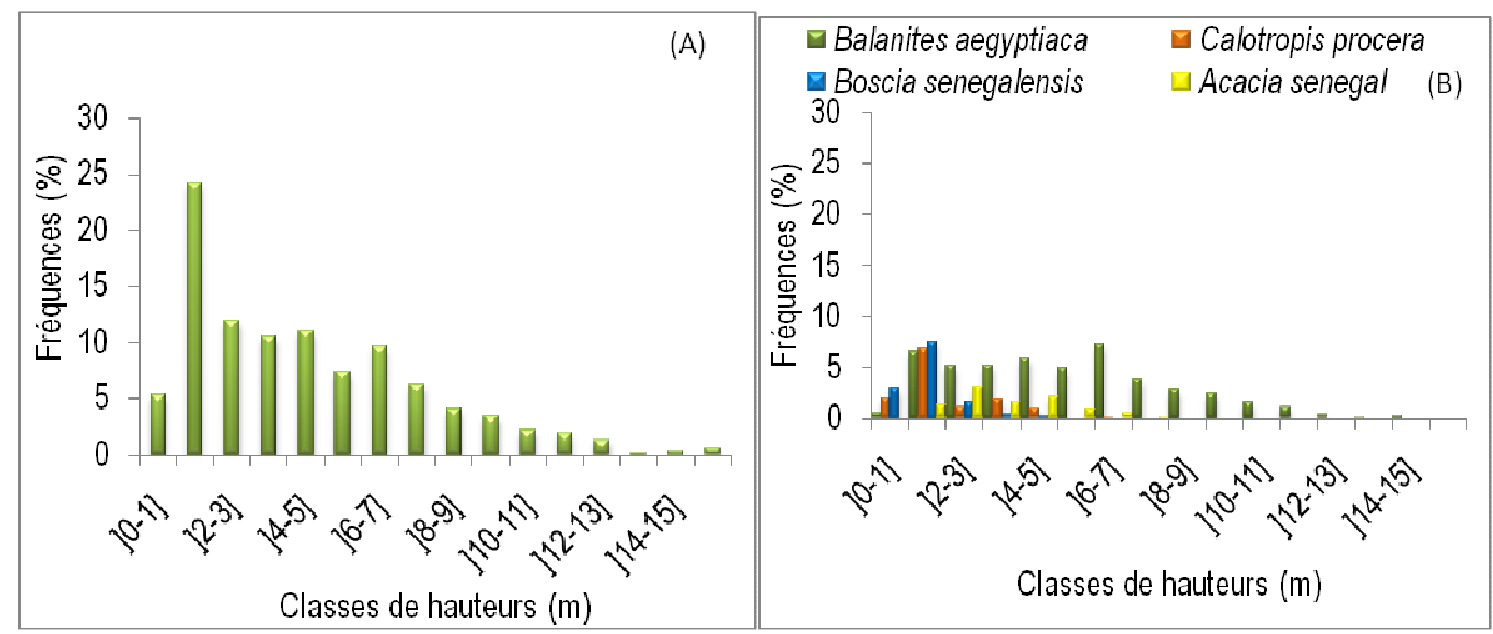

Figure 2 : Distribution selon la hauteur du peuplement et des individus dominants des quatre sites

Environ $61 \%$ des individus de la zone étudiée ont une hauteur inférieure à $5 \mathrm{~m}$ tandis que moins de $20 \%$ seulement dépassent $7 \mathrm{~m}$ de hauteur (Fig. 2A). La distribution selon la hauteur révèle donc une hétérogénéité dans la structure verticale de ce peuplement. L'essentiel des individus de petite taille du peuplement appartient à $B$. aegyptiaca, $B$. senegalensis et $C$. procera. Ces deux dernières sont des arbustes de taille souvent comprise entre 1 et $2 \mathrm{~m}$ (fig. 2B). II faut aussi noter la présence d'A. senegal qui sont généralement des arbustes de moins de $7 \mathrm{~m}$ de hauteur (Fig. 2B). Seuls les individus de $B$. aegyptiaca dépassent $7 \mathrm{~m}$ de hauteur (Fig. 2B). La structure horizontale illustrée par la distribution selon la circonférence des individus du peuplement $(A)$ et des quatre espèces dominantes $(B)$ est représentée par la figure 3 . 


\section{Niang et al. J. Appl. Biosci. 2014. Flore et structure de la vegetation ligneuse le long de la Grande}

Muraille Verte au Ferlo, nord Senegal

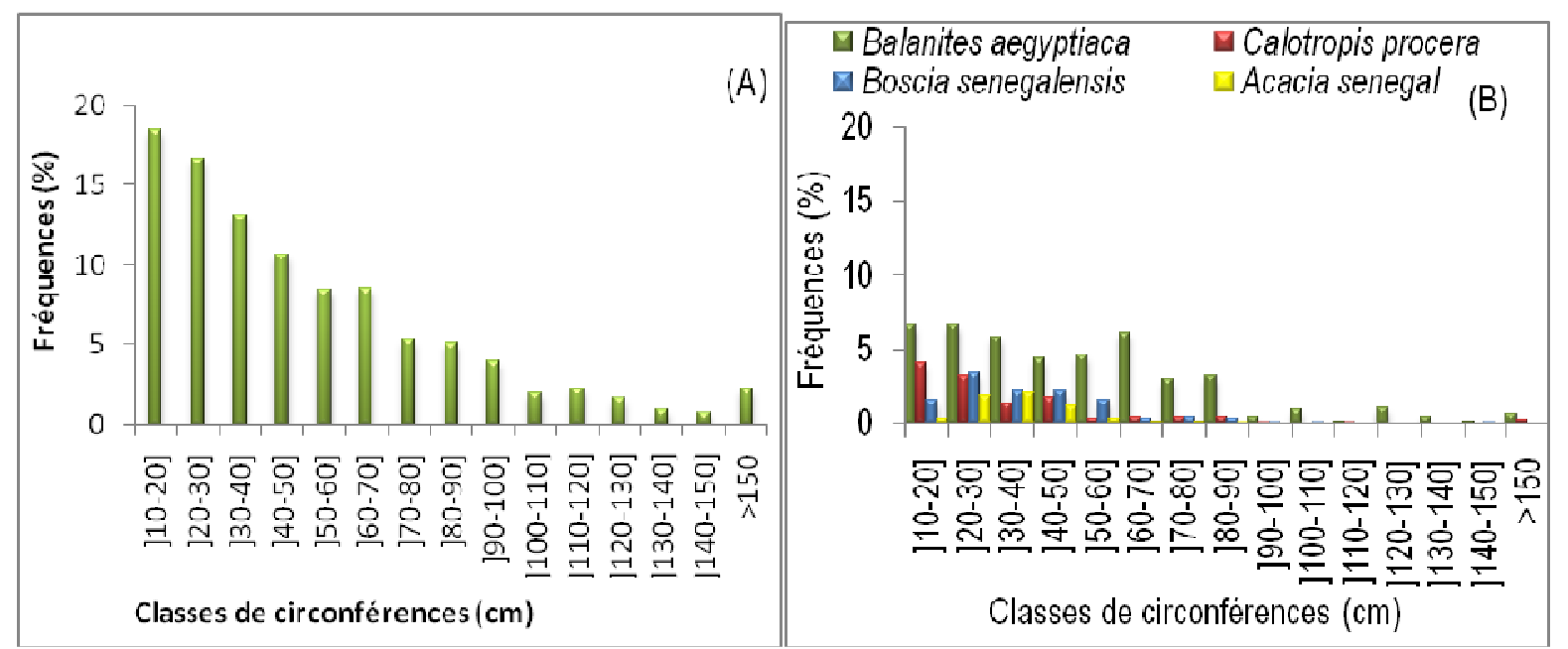

Figure 3 : Distribution selon la circonférence des individus dominants des quatre sites

Les individus du peuplement sont distribués dans toutes les classes de circonférences. Cependant, près de $60 \%$ des individus ont une circonférence inférieure à $50 \mathrm{~cm}$ (Fig. 3A). Le peuplement peut donc être considéré comme étant très jeune ou du moins constitué pour l'essentiel par des arbustes et que la structure horizontale est également hétérogène. Ces individus de petite circonférence appartiennent généralement à quatre espèces (B. aegyptiaca, $C$. procera, $B$. senegalensis et $A$. senegal) (Fig. 3B). Al'image de la distribution selon la hauteur, seule l'espèce $B$. aegyptiaca renferme des individus de plus de $100 \mathrm{~cm}$ de circonférence (Fig. 3B).
Régénération : La figure 4 montre la distribution des jeunes plantes suivant les sites et les différentes espèces du peuplement. Widou $(40,79 \%)$ présente la plus importante régénération, il est suivi de Tessékéré $(22,78 \%)$ et de Lougré $(21,22 \%)$. La station de Labgar $(10,20 \%)$ présente le plus faible taux de régénération (Fig. 4A). B. aegyptiaca (64\%) est l'espèce qui régénère mieux, elle est de loin suivie de $B$. senegalensis et de $C$. procera avec des taux respectifs de 18 et 7,69\%. Les autres espèces présentent un taux de régénération très faible (Fig. 4B).

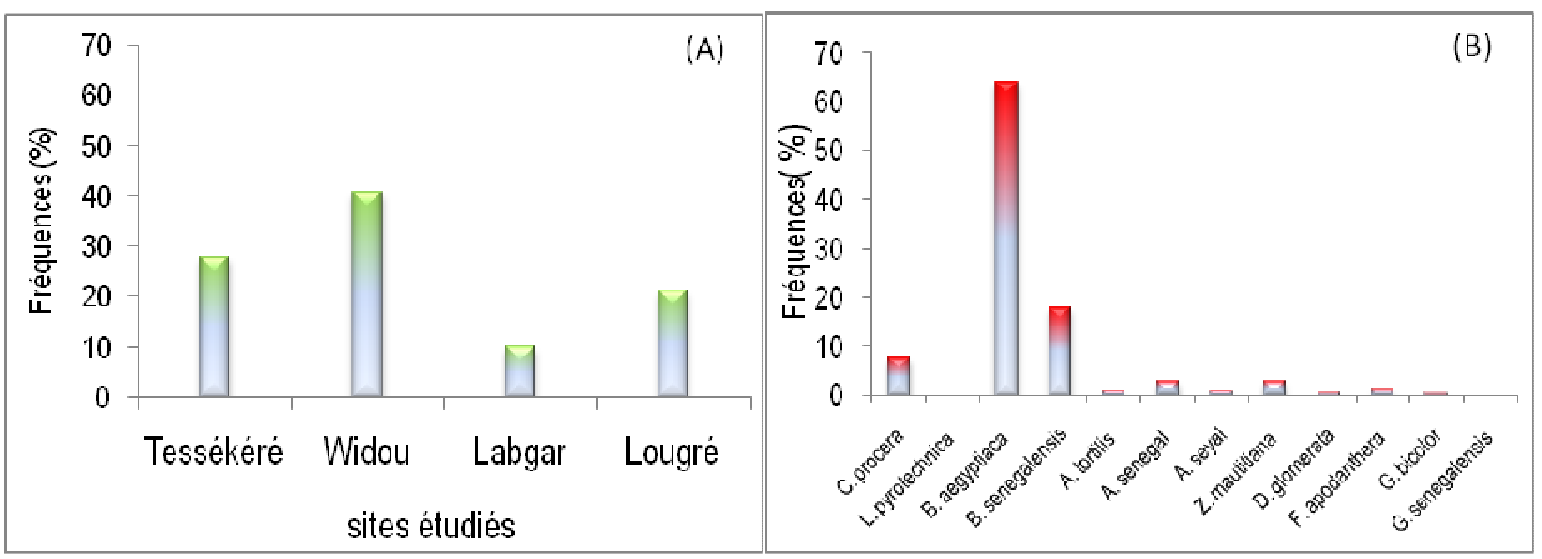

Figure 4. Distribution des jeunes plantes au niveau des différents sites étudiés et celles des espèces recensées.

Recouvrement : Le couvert aérien varie de $1959,9 \mathrm{~m}^{2} / \mathrm{ha}$ à $712,56 \mathrm{~m}^{2} /$ ha soit de $12,57 \%$ à $9,34 \%$ d'un site à l'autre. II est plus important à Widou $\left(1959,9 \mathrm{~m}^{2} / \mathrm{ha}\right)$ et Tessékéré $\left(1257,06 \mathrm{~m}^{2} / \mathrm{ha}\right)$ et faible à Lougré $(934,8$ $\mathrm{m}^{2} / \mathrm{ha}$ ) et Labgar $\left(712,56 \mathrm{~m}^{2} / \mathrm{ha}\right)$. A Tessékéré, la surface terrière est de $3,52 \mathrm{~m}^{2} /$ ha, répartie principalement entre
A. digitata $\left(1,27 \mathrm{~m}^{2} / \mathrm{ha}\right)$, B. aegyptiaca $\left(0,61 \mathrm{~m}^{2} / \mathrm{ha}\right)$ et C. procera $\left(0,57 \mathrm{~m}^{2} / \mathrm{ha}\right)$. Elle est plus importante à Widou où elle est de $4,35 \mathrm{~m}^{2} / \mathrm{ha}$. Ce recouvrement basal est de $3,19 \mathrm{~m}^{2} /$ ha à Lougré. A Labgar, la surface terrière est de $2,5 \mathrm{~m}^{2} / \mathrm{ha}$; elle est répartie entre B.aegyptiaca $(1,07$ $\mathrm{m}^{2} / \mathrm{ha}$ ) et $D$. melanoxilon $\left(1,09 \mathrm{~m}^{2} / \mathrm{ha}\right)$. 


\section{Niang et al. J. Appl. Biosci. 2014. Flore et structure de la vegetation ligneuse le long de la Grande}

Muraille Verte au Ferlo, nord Senegal

\section{DISCUSSION}

La présente étude révèle que la flore ligneuse des parcours communautaires, le long du tracé de la Grande Muraille Verte, est riche de 20 espèces réparties entre 18 genres et 12 familles. Comparée aux résultats de Diouf et al. (2002) qui ont travaillé dans la zone sahélienne plus précisément au Ferlo-Nord avec 11 espèces réparties en 9 genres et 8 familles, elle est plutôt riche. Cependant comparée à ceux de Ndiaye (2008) travaillant dans la zone sahélienne, les résultats sont quasi-similaires; ce dernier avait recensé 23 espèces réparties entre 18 genres et 13 familles. Ainsi nous avons un enrichissement de cette flore depuis les observations de Diouf et ses collaborateurs jusqu'en 2008 et un léger appauvrissement par la suite. Cette dégradation des ressources du Ferlo se confirme avec les travaux de Diallo et al. (2011) et ceux de Ndiaye (2013). L'analyse des fréquences de présence des espèces dans les différents sites permet d'établir leur étendue de distribution ainsi que leur degré d'ubiquité ou de spécificité (Le Bourgeois et Guillerm, 1994). Ainsi trois groupes d'espèces sont distingués selon leurs fréquences de présence dans les différents sites: les espèces très fréquentes qui sont souvent rencontrées dans tous les sites, les espèces fréquentes, présentes au maximum dans trois sites et les espèces peu fréquentes ou rares, rencontrées dans un seul site: $A$. obesum, $C$. glutinosum, G. senegalensis, L. pyrotechnica, K. africana, $D$. glomerata, $P$. chilensis, $D$. melanoxilon et $P$. lucens. Ce sont généralement des espèces caractéristiques qui révèlent par leur présence une spécificité écologique $d u$ biotope (Akpo, 1998). L'importance de la surface terrière est corrélée à la présence et à la fréquence des individus à gros tronc comme $A$. digitata et $B$. aegyptiaca. Cela s'explique par le fait que ces espèces disposent d'un diamètre très élevé alors que la surface terrière est fonction du diamètre (Konaté, 1999; Ndiaye, 2013). L'importance du couvert végétal au Ferlo s'explique par l'aire de projection des espèces dominantes du peuplement. En effet, ces espèces sont à cimes étalés plus ou moins ouvertes. B. aegyptiaca, C. procera, $B$. senegalensis, A. senegal et $A$. tortilis sont les espèces dominantes, elles offrent ainsi leur physionomie au peuplement ligneux. Elles sont le plus souvent, mieux adaptées aux conditions climatiques et édaphiques du Sahel (Konaté, 1999). La sclérophyllie élevée de la plupart de ces espèces serait un caractère de xéromorphose et favoriserait leur développement (Vanpraet et Itterum, 1983; Poupon, 1980; Broutin et
Sokano, 1992). Des espèces répandues comme $A$. tortilis sont de mauvais indicateurs des conditions pédoclimatiques (Ganaba et Guinko, 1995). D'autres espèces comme $S$. birrea, $C$. glutinosum, $A$. senegal ont été particulièrement atteintes par la sécheresse persistante des deux dernières décennies (Miehe-Klug, 1990) et par une forte anthropisation (Diop, 1989), ce qui serait à l'origine de leur régression. La substitution de $A$. tortilis par $C$. procera et la persistance de $B$. senegalensis s'explique par leur capacité d'adaptation plus grande due à un degré de sclérophyllie élevée pour Boscia senegalensis (Broutin et Sokano, 1992), de même l'utilisation fréquente des fruits de $A$. tortillis pour nourrir le bétail impliquant une diminution de sa densité. Cette situation traduise une dégradation du milieu qui se manifeste par une diminution de la densité du peuplement et l'accroissement de la distance moyenne entre individus (Diouf et al., 2002; Diallo et al., 2011). La régénération est importante chez $B$. aegyptiaca et très faible chez les autres espèces dont la densité était élevé autrefois. C'est le cas de A. senegal (Diallo et al., 2013), A. tortilis, S. birrea, A. seyal, C. glutinosum, Z. mauritiana, $A$. leiocarpa et $G$. bicolor. La faible régénération de ces espèces peut s'expliquer par plusieurs facteurs selon Boussim et Gampine (1995) et Ndiaye (2013). Selon Konaté (1999), l'abondance de la régénération d'une espèce serait due à son adaptabilité aux conditions pédoclimatiques et à son utilisation moins importante par les populations. L'importance de la régénération de $B$. aegyptiaca et de $B$. senegalensis peut être aussi liée à leur sclérophylle élevée (Poupon, 1980). Selon Diouf et al. (2002), la régénération élevée de $B$. aegyptiaca s'explique par son comportement hydrique et photosynthétique. En effet, $B$. aegyptiaca est un ligneuxà épines et rameaux photo-synthétiquement actifs toute l'année et à feuilles réduites. L'importance de la régénération de $B$. aegyptiaca peut également s'expliquer par la propagation des graines via les hommes et/ou les animaux (Broutin et Sokona, 1992), sa dissémination peut se faire par endozoochorie (Akpo et al., 1995). De plus, le couvert aérien de $B$. aegyptiaca est privilégié par les ruminants et cette fréquentation augmente les chances de dissémination des semences qui y trouvent par ailleurs des conditions favorables à leur implantation (Akpo et Grouzis, 1996). La faible régénération des espèces comme $A$. senegal s'explique par l'intérêt qu'il présente pour le bétail et la gomme (Diallo, 2007; Diallo, 2013). 


\section{CONCLUSION}

Au cours de cette étude, une vingtaine d'espèces appartenant à 18 genres et 12 familles ont été recensée. Certaines espèces comme $B$. aegyptiaca, $A$. digitata, $A$. seyal et $A$. tortilis sont communes à toutes les stations. Cependant d'autres comme C. procera, B. senegalensis, A. senegal, $C$. glutinosum, ne sont retrouvées que dans certains sites. Les caractéristiques structurales ainsi observées permettent d'affirmer que la végétation ligneuse est de plus en plus dégradée sous l'action simultanée des péjorations climatiques et de l'anthropisation croissante. Cette situation va nécessiter la mise en place de projets et des techniques adaptés pour la reconstitution des écosystèmes et la préservation des ressources végétales indispensables pour une zone dont l'élevage constitue l'activité économique principale.

\section{REMERCIEMENTS}

Nous remercions l'Observatoire Homme-Milieu (OHMi) Tessékéré et l'institut Klorane pour leur appui à la réalisation de ce travail.

\section{REFERENCES BIBLIOGRAPHIQUES}

Akpo LE, 1998. Effet de l'arbre sur la végétation herbacée dans quelques phytocénoses au Sénégal. Variation selon un gradient climatique. Thèse de doctorat d'état en Sciences Naturelles, Univ. Cheikh Anta Diop, Dakar, 61, 133 p.

Akpo LE, Grouzis M, Bâ AT, 1995. L'arbre et l'herbe au sahel: effet des arbres sur la composition minérale des pâturages naturels du NordSénégal (Afrique de l'Ouest). Revue Med.Vet.6, 663-670.

Akpo LE. et Grouzis M,1996. Influence du couvert sur la régénération de quelques espèces ligneuses sahéliennes (Nord Sénégal, Afrique occidentale). Webbia50(2): 247-263.

Asecna, 2008. Paramètre météorologique relatifs à la période 1997-2006. Dakar-Sénégal. Fiche de relevés climatiques $1 \mathrm{p}$.

Boudet G, 1984. Manuel sur les pâturages tropicaux et les cultures fourragères. 4ème édition. Paris, Ministère de la Coopération, Manuel et Précis d'élevage 4, $254 \mathrm{p}$.

Boussim Jl. et Gampine D, 1995. Étude des contraintes à la régénération naturelle de quelques espèces locales de Combretaceae et de Caesalpiniaceae au Burkina-Faso. Études sur la Flore et la végétation du Burkina Faso et des pays avoisinants, Vol II p. 33 - 41.

Broutin C. et Sokona K, 1992. La production d'huile de Sump dans la zone sylvo-pastorale du nord du Sénégal. Rapport, $34 \mathrm{p}$.

Diallo A, 2007. Dynamique des populations d'Acacia senegal dans quelques forêts du Sénégal. Ropport, $10 \mathrm{p}$.

Diallo A, Faye MN, Guissé A, 2011. Structure des peuplements ligneux dans les plantations d'Acacia senegal (L.) Willd dans la zone de
Dahra (Ferlo, Sénégal) Rev. Écol. (Terre Vie), vol. 66, 2011.

Diallo A, Agbangba EC, Ndiaye O, Guissé A, 2013. Ecological Structure and Prediction Equations for Estimating Tree Age, and Dendometric Parameters of Acacia senegal in the Senegalese Semi-Arid Zone -Ferlo.American Journal of Plant Sciences, 2013, 4, 1046-1053.

Diop AT, 1989. Les ressources ligneuses de la zone sylvo-pastorale du Sénégal: évolution, gestion et perspectives de développement. Communication, $13 \mathrm{p}$.

Diouf M, Akpo LE, Rocheteau A, Do F, Goudiaby V, Diagne AL, 2002. Dynamique du peuplement ligneux d'une végétation sahélienne au NordSénégal (Afrique de l'ouest). J.Sc.Vol. $2 \mathrm{~N}^{\circ} 1.10$ p.

Faye G, Frison PL, Wade S, Ndione JA, Beye AC, Rudant JP, 2011. Étude de la saisonnalité des mesures des diffusiomètres scat : apport au suivi de la végétation au sahel, cas du Ferlo au Sénégal. Rev Télédétection, vol. 10, n 1, p. 2331.

Ganaba S. et Guinko S, 1995. Morphologie et rôle des structures racinaires dans la mortalité de Pterocarpus lucens Lepr. dans la région sahélienne de la mare d'Oursi (Burkina Faso). Études sur la Flore et la végétation du Burkina Faso et des pays avoisinants Vol. II p15-24.

Grouzis M. et Albergel J, 1989. Du risque climatique à la contrainte écologique: incidence de la sécheresse sur les productions végétales et le milieu au Burkina Faso. In "Le risque en agriculture », Eldin M., Milleville P. Edition, Collection à travers Champs, ORSTOM, Paris, 243-254 p. 


\section{Niang et al. J. Appl. Biosci. 2014. Flore et structure de la vegetation ligneuse le long de la Grande}

Muraille Verte au Ferlo, nord Senegal

Grouzis M. et Albergel J, 1991. Du risque climatique à la contrainte écologique: Incidences de la sécheresse sur les productions végétales et le milieu au Burkina-Faso. In: ELDIN et MILLEVILL (EEds.), (Le risque en agriculture 243-254, ORSTOM éd., Paris 620 p.).

Konaté PS,1999. Structure, composition et distribution de quelques ligneux dans les provinces du Seno et du Yagha: proposition d'application à leur gestion. Mém. de fin d'études d'ingénieur du développement rurale, $76 p$.

Le Bourgeois TH. et Guillerm JL, 1994. Étendue de distribution et degré d'infestation des adventices dans la rotation cotonnière au Nord-Cameroun. WEEDResearch, 1995. Vol. 35 : 89-98.

Le Houerou HN, 1980. Le rôle des ligneux fourragers dans les zones sahélienne et soudaniennes. CIPEAIALCA, actes du colloque sur les fourrages ligneux en Afrique, Addis-Abeba, Avril $1980,85-101$.

Matern B, 1956. On the geometry of the cross section of a stem. Medd-stat. Skogsforsk-ninsings. 46, 128.

Miehe-klug S,1990. Inventaireetsuivi (Monitoring) dans la parcelled'élevage à WidduThiengoly (Ferlo Senegal).GTZ, Hamburg, $114 \mathrm{p}$.

Ndiaye I, 2008. Flore et végétation ligneuses du terroir de Katané dans la réserve de faune du Ferlo-nord. Mém. de DEA, FST, $25 \mathrm{p}$.

Ndiaye O., 2013. Caractéristiques des sols, de la flore et de la végétation du Ferlo sableux, Nord Sénégal. Thèse unique. $124 \mathrm{p}$.

Niang K, 2009. L'arbre dans les parcours communautaires du Ferlo (Nord-Sénégal). Mém. De DEA. 69 p.

P.O.D.E.S., 2004. Xéme plan d'orientation pour le développement économique et social 20022007. «Croissance forte et durable, réduction de la pauvreté et bonne gouvernance ».Ministère du plan; Direction de la planification nationale et de la coordination avec la planification régionale. Projet de $214 \mathrm{p}$.

Poupon H. et Bille JC, 1974. Recherches écologiques sur une savane sahélienne du Ferlo septentrional, Sénégal. Influence de la sécheresse de l'année 7372-1973 sur la strate ligneuse. Revue Ecol. (Terre et Vie) 28:49-75.

Poupon $\mathrm{H}, 1980$. Structure et dynamique de la strate ligneuse d'une steppe sahélienne au Nord du Sénégal-ORSTOM, Paris, $351 \mathrm{p}$.
Ramade F, 1990. Éléments d'écologie. Écologie fondamentale. Mc GRAW-HILL., Paris, 403 p.

Sharman MJ. et Gning M, 1983. Comportement du cheptel au Ferlo. Résultats des suivis quotidiens (Com. Coll. sur méthodes d'inventaire et de surveillance continu des écosystèmes pastoraux sahéliens. Application de développement. Dakar, 16, 17, 18 novembre 1983): PP: 209221.

Toupet C, 1989. Comparaison des sécheresses historiques et de la sécheresse actuelle: essai de définition de ka sécheresse et de l'aridification. In: BRET coord.: Les hommes face aux sécheresses, Nordeste brésilien, Sahel africain: 77- 84, EST- IHEAL éd. (422 pp.).

Toutain B, Bortoli L, Dulieu D, Forgiarini G, Menaut JC, Piot $J$, 1983. Espèces ligneuses et herbacées dans les écosystèmes sahéliens pâturés de Haute-Volta, ACC GRIZA (LAT), GERDAT, 124 p.

Vanpraet CL. et Itterum,1983. Considérations sur des analyses dimensionnelles de quelques espèces ligneuses de la zone sylvo-pastorale au Sénégal (Com, Coll. sur méthodes d'inventaire et de surveillance continue des écosystèmes pastoraux sahéliens. Application au développement, Dakar, 16, 17, 18 novembre 1983) - pp: 243-249. 